\title{
Network Analysis Based on Unique Spectral Features Enables an Efficient Selection of Genomically Diverse Operational Isolation Units
}

\author{
Charles Dumolin ${ }^{1} \mathbb{D}$, Charlotte Peeters ${ }^{1}$, Evelien De Canck ${ }^{1}$, Nico Boon ${ }^{2} \mathbb{D}$ and Peter Vandamme ${ }^{1, *(\mathbb{D})}$ \\ 1 Laboratory of Microbiology, Department of Biochemistry and Microbiology, Faculty of Sciences, \\ Ghent University, BE-9000 Ghent, Belgium; Charles.Dumolin@UGent.be (C.D.); \\ Charlotte.Peeters@UGent.be (C.P.); Evelien.DeCanck@UGent.be (E.D.C.) \\ 2 Center for Microbial Ecology and Technology (CMET), Department of Biotechnology, Faculty of Bioscience \\ Engineering, Ghent University, BE-9000 Ghent, Belgium; Nico.Boon@UGent.be \\ * Correspondence: Peter.Vandamme@UGent.be
}

Citation: Dumolin, C.; Peeters, C.; De Canck, E.; Boon, N.; Vandamme, P. Network Analysis Based on Unique Spectral Features Enables an Efficient Selection of Genomically Diverse Operational Isolation Units. Microorganisms 2021, 9, 416. https://doi.org/10.3390/ microorganisms 9020416

Academic Editor: Nelson Lima

Received: 8 January 2021

Accepted: 13 February 2021

Published: 17 February 2021

Publisher's Note: MDPI stays neutral with regard to jurisdictional claims in published maps and institutional affiliations.

Copyright: (c) 2021 by the authors. Licensee MDPI, Basel, Switzerland. This article is an open access article distributed under the terms and conditions of the Creative Commons Attribution (CC BY) license (https:// creativecommons.org/licenses/by/ $4.0 /)$.

\begin{abstract}
Culturomics-based bacterial diversity studies benefit from the implementation of MALDITOF MS to remove genomically redundant isolates from isolate collections. We previously introduced SPeDE, a novel tool designed to dereplicate spectral datasets at an infraspecific level into operational isolation units (OIUs) based on unique spectral features. However, biological and technical variation may result in methodology-induced differences in MALDI-TOF mass spectra and hence provoke the detection of genomically redundant OIUs. In the present study, we used three datasets to analyze to which extent hierarchical clustering and network analysis allowed to eliminate redundant OIUs obtained through biological and technical sample variation and to describe the diversity within a set of spectra obtained from 134 unknown soil isolates. Overall, network analysis based on unique spectral features in MALDI-TOF mass spectra enabled a superior selection of genomically diverse OIUs compared to hierarchical clustering analysis and provided a better understanding of the inter-OIU relationships.
\end{abstract}

Keywords: MALDI-TOF MS; network cluster analysis; bacterial diversity; species subgrouping

\section{Introduction}

In the last decade, there has been a revived interest in the cultivable bacterial diversity from various ecosystems. Microorganisms feature a countless repertoire of metabolites and enzymes with industrial potential [1]. In 2015, the global market for microbes and microbial products was estimated at $\$ 186.3$ billion and had an expected compound annual growth rate of $10.2 \%$ up to 2023 [2]. The development of lean but highly diverse microbe libraries permits efficient industrial exploitation [3]. In addition, the description and differentiation of both species and strains within a species are of equal importance to environmental monitoring, clinical microbiology, as well as water quality and food safety $[4,5]$. However, long-term maintenance of large culture collections and processing of genomically redundant isolates result in an exponential increase in analysis costs.

Lagier and colleagues [6] showed that exhaustive isolation campaigns are complementary to metagenomic techniques for the description of bacterial diversity. In these so-called culturomics studies, the use of Matrix-Assisted Laser Desorption/Ionization Time-of-Flight Mass-Spectrometry (MALDI-TOF MS) is crucial for fast and accurate dereplication and identification of isolates. Commercial MALDI-TOF MS-based bacterial identification systems nowadays come with a comprehensive database to identify a query isolate at genus or species level $[7,8]$. However, to date, these databases are restricted to mainly clinical and food-related organisms and include a limited number of microorganisms from other ecosystems $[9,10]$. It is preferable that dereplication, i.e., the process of reducing a set of isolates based on similarity into a set of non-redundant strains, is not limited to species 
level differentiation because variation in a species accessory genome determines functional properties as well [5,11-13]. Obtaining this level of dereplication resolution in culturomics studies is a challenging task and requires an approach that facilitates to recognize subtle peak differences among strains of the same species [14].

We recently described a novel algorithm, SPeDE, specifically developed for the dereplication of large MALDI-TOF MS spectral datasets at the infraspecific level [15]. In contrast to database-driven approaches, the algorithm analyzes spectra in a pairwise manner and defines spectral differences as unique spectral features (USFs). This principle resembles the detection of specific biomarker peaks, which allows differentiation of spectra at a higher resolution [14,16]. Based on a USF matrix, a set of non-redundant reference spectra is selected, and each reference spectrum represents an operational isolation unit (OIU) of matched spectra. Validation of the algorithm with multiple datasets showed that these OIUs reflect the intraspecific diversity examined by differentiating MALDI-TOF mass spectra of bacterial strains sharing a minimum of $98 \%$ genome-wide average nucleotide identity (ANI) [15]. Thus, constructing bacterial collections by retaining only one representative isolate (i.e., a reference) for each OIU facilitates the selection of genomically distinct isolates within an isolate collection. The SPeDE algorithm, however, does not provide a tool to estimate the degree of similarity between references which could be used to further reduce the number of isolates for downstream analyses. The tool described in the present study aims to prioritize the elimination of less valuable OIUs such as genomically redundant OIUs induced by biological or technical sample variation.

Hierarchical clustering is well established to group spectra based on their similarity, but their taxonomic resolution appears to be less reliable the more closely related clusters are [17-19]. Network analysis is a tool frequently used to explore associations between known and unknown components in MS/MS data [20,21]. In the present study, we compared hierarchical clustering and network analysis as visualization tools to assess the relatedness between references based on the USF matrix obtained from the SPeDE algorithm.

\section{Materials and Methods}

\subsection{Lactobacillus Brevis and Benchmark MALDI Datasets}

Publicly available MALDI-TOF MS data of a L. brevis dataset and spectra of Burkholderia strains of a benchmark dataset were taken from a previous study (Supplementary Table S1) [15].

\subsection{Isolation, MALDI-TOF MS Sample Preparation and Data Acquisition}

A soil sample of the Almoeseneie forest (Gontrode, Belgium, $50^{\circ} 58^{\prime} \mathrm{N} 3^{\circ} \mathrm{E} 49^{\prime}$ ) [22] was plated onto 1/100 diluted nutrient broth (Oxoid, Basingstoke, UK) supplemented with $\mathrm{MgSO}_{4} .7 \mathrm{H}_{2} \mathrm{O}(2 \mathrm{mM})$, cycloheximide $(0.035 \mathrm{mM})$, and 3-(N-morpholino) propanesulfonic acid (MOPS, $5 \mathrm{mM}$ ). The $\mathrm{pH}$ was set at 7 using $20 \% \mathrm{KOH}(\mathrm{w} / \mathrm{v})$, and the nutrient broth was solidified with Gelzan TM CM (Sigma-Aldrich, St.-Louis, MO, USA, $10 \mathrm{~g} \mathrm{~L}^{-1}$ ). After 25 days of incubation at $20^{\circ} \mathrm{C}$ in the dark, colonies were picked and transferred to 96 -well plates containing 1/100 diluted nutrient broth. After 10 days of incubation, the cultures were subcultivated using a Viaflo 96/384 pipetting robot (Integra, Le Locle, Switzerland) to 96-well deep well plates containing $0.8 \mathrm{~mL}$ of $1 / 10$ diluted nutrient broth (Oxoid, Basingstoke, UK) supplemented with MOPS (5 mM) and set to a final $\mathrm{pH}$ of 7.0.

After 10 days of incubation, $100 \mu \mathrm{L}$ of each deep well plate cultures were transferred to a novel 96-well plate containing $10 \mu \mathrm{L}$ glycerol (Sigma-Aldrich, St.-Louis, MO, USA) for storage of the cultures at $-80{ }^{\circ} \mathrm{C}$. The remaining cell suspension was used for semiautomated MALDI-TOF MS-sample preparation by using a Viaflo 96/384 pipetting robot (Integra, Le Locle, Switzerland) as described earlier [15]. Cell suspensions (3 $\mu \mathrm{L})$ were spotted on a target plate (Bruker Daltonik, Bremen, Germany) in duplicate. The sample spot was overlaid with $1 \mu \mathrm{L}$ of matrix solution $\left(10 \mathrm{mg} \mathrm{mL}^{-1} \alpha\right.$-cyano-4-hydroxycinnamic acid in acetonitrile:water:trifluoroacetic acid 50:47.5:2.5). Subsequently, MALDI-TOF MS profiles were acquired on the Bruker MicroflexTM LT/SH (Bruker Daltonik, Bremen, Germany) as previously described [15]. Spectra were compared to the Bruker MBT (version DB 
5989) using MBT Compass Explorer according to manufacturer's settings (Bruker Daltonik, Bremen, Germany). Identification scores were considered of 'high-confidence' $(\geq 2.00)$, 'low-confidence' (1.70-2.00) or 'no identification possible' $(<1.70)$. A total of 136 spectra were identified as belonging to the genus Burkholderia with high or low-confidence scores (data not shown) and were retained for further analysis. Subsequently, 64 additional spectra that remained unidentified exhibited no USFs with some of these 136 Burkholderia spectra, as revealed by applying the SPeDE algorithm (see below). They were therefore included in the subsequent analyses as well. The final dataset of the present study therefore consisted of 200 spectra which derived from 134 isolates (i.e., 66 isolates were represented by two technical replicate spectra) (Supplementary Table S1).

\subsection{SPeDE Dereplication, Hierarchical Clustering and Network Analysis}

All datasets were processed with the SPeDE algorithm using default parameters for peak accuracy window (50) and local PPMC (700) [15].

To generate a dendrogram, the USF matrix of references was processed using the Jupyter notebook provided on the SPeDE GitHub account (https://github.com/LMUGent/SPeDE, accessed on 17 April 2019), after which it was exported to a Newick format and processed further with iTol [23]. A dendrogram was derived from the unweighted pair group method with arithmetic mean (UPGMA) with levels of linkage expressed as the relative distance in observed USFs [15].

To generate the network, the USF matrix was converted to the three-column stack format indicating for each pair of spectra how many USFs were detected in one direction [24]. This matrix was filtered to retain only combinations for which zero USFs were detected. Based on this final matrix the interactive network was generated using the Python packages NetworkX and Bokeh [24,25]. In the network, each node represents a spectrum and the edges (connecting lines between nodes) represent all combinations for which no USF could be detected. The code to generate the graph is available in a Jupyter notebook at https: / github.com/LM-UGent/ $\mathrm{SPeDE} /$ tree/master/output_network (accessed on 23 December 2020).

\subsection{Genome Sequencing, Assembly and Analysis of 14 Case Study Isolates}

DNA was extracted using an automated Maxwell ${ }^{\circledR}$ DNA preparation instrument (Promega, Madison, WI, USA). The final extract was treated with RNase $\left(2 \mathrm{mg} \mathrm{mL}^{-1}, 5 \mu \mathrm{L}\right.$ per $100 \mu \mathrm{L}$ extract) and incubated at $37^{\circ} \mathrm{C}$ for one hour. DNA quality was checked using 1\% agarose gel electrophoresis and DNA quantification was performed using the QuantiFluor ONE dsDNA system and the Quantus fluorometer (Promega, Madison, WI, USA). Afterwards, DNA was stored at $-20{ }^{\circ} \mathrm{C}$ before further analysis. Paired-end $2 \times 150$ bp libraries were prepared at the Wellcome Trust Human Genome Center (Oxford, Basingstoke, UK) using the NEBNext DNA library kit for Illumina (New England Biolabs, Ipswich, MA, USA) and sequenced on an Illumina HiSeq 4000 instrument. De novo assembly was performed with Shovill pipeline v1.0.0 (https:/ / github.com/tseemann/shovill, accessed on 4 December 2018). The QUAST v4.0 program (19 April 2016).was used to generate the summary statistics of the assembly (N50, maximum contig length, GC) [26]. Pairwise average nucleotide identity (ANI) was calculated using OrthoANI software v0.90 (14 April 2017) [27].

\section{Results}

\subsection{Network Analysis Clustered OIUs Representing the Same L. brevis Strains Together}

Previously, a set of 549 spectra derived from 25 L. brevis strains was used to test for the robustness of the SPeDE dereplication algorithm towards biological and technical sample variation [15]. SPeDE dereplication of the 549 spectra resulted in 35 operational isolation units (OIUs) and yielded at least one OIU reference for 24 of the 25 studied strains (Supplementary Table S2). Seven (i.e., LMG 11438, LMG 11495, LMG 11988, LMG 12023, LMG 18022, R-47325 and R-49154) and two (LMG 11969 and R-42874) strains were represented by two and three references, respectively [15]. 
In the present study, we performed hierarchical clustering analysis of the OIU references which visualized the relative distance in the number of USFs observed between these references (Figure 1A). For four strains with multiple references (LMG 11988, LMG 18022, R-42874 and R-47325), the references clustered close to each other. The references of the remaining five strains with multiple references (LMG 11438, LMG 11495, LMG 11969 and R-49154) did not cluster together. Thus, hierarchical clustering of the references did not group genomically redundant OIUs obtained from the same L. brevis strains due to biological or technical sample variation [15].
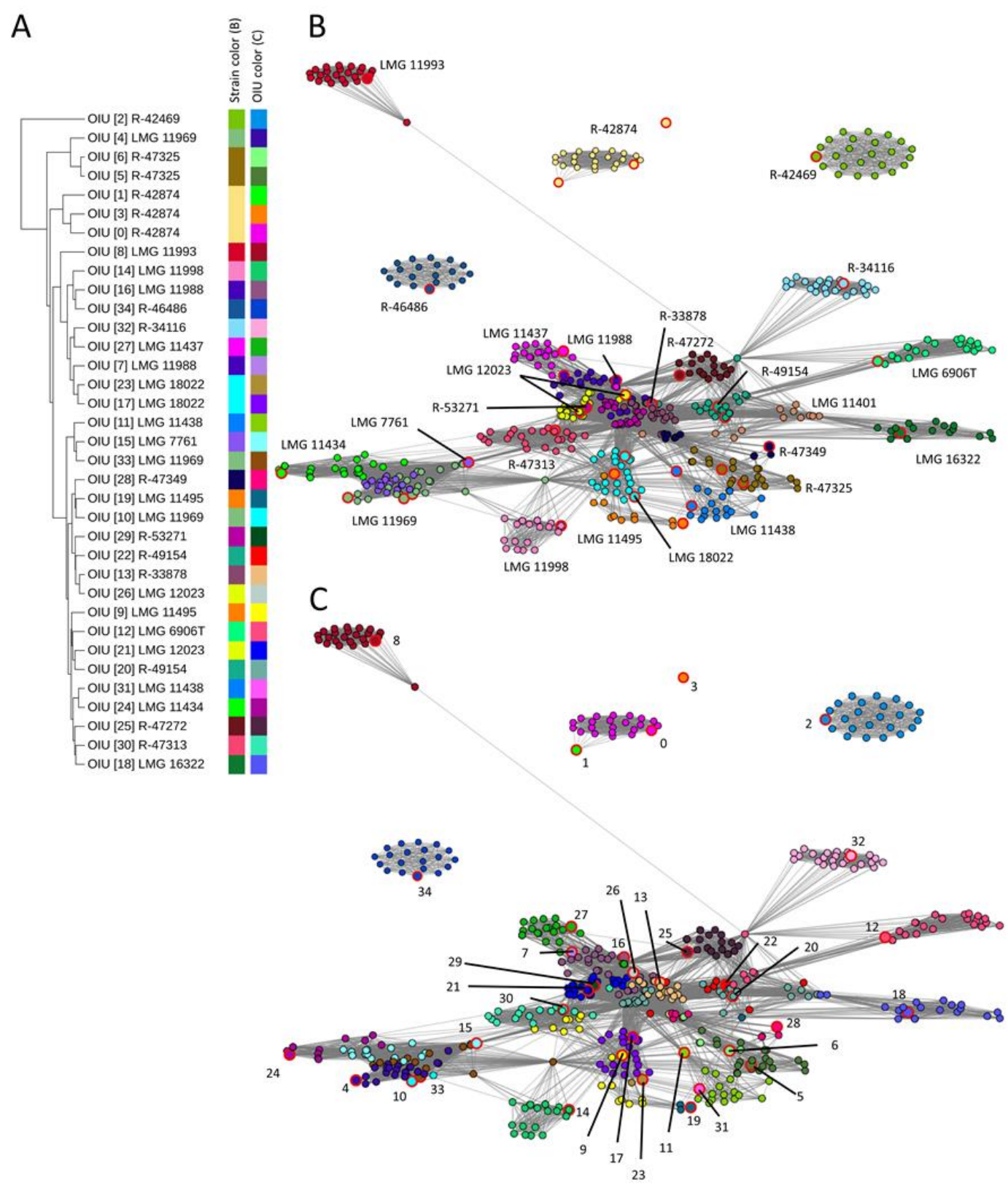

Figure 1. Comparison of hierarchical clustering versus network analysis of the L. brevis dataset. (A) Hierarchical clustering dendrogram of 35 reference spectra (UPGMA algorithm) using the relative distance in the number of detected USFs. Color bars depict strains and OIUs. (B) Network analysis based on all zero USFs elements (edges) of the USF matrix. The individual nodes are colored according to the strain number, as depicted in panel (A). (C) Network analysis based on all zero USFs elements (edges) of the USF matrix. Individual nodes are colored according to OIU number, as depicted in panel (A). The reference spectrum of each OIU is highlighted with large node sizes and red marking (B,C). 
Subsequently, a network was generated based on the USF matrix that visualized all matrix elements between which no USFs could be detected (value $=0$ ). These elements were shown in the network as a connection (edge) in between the spectra (represented as nodes). The network nodes were color coded according to either their corresponding strain identity (Figure 1B) or the OIU classifier as generated by the SPeDE algorithm (Figure 1C). In the network, all references corresponding to the same strain were grouping near each other (Figure 1B), except for one reference of strain R-42874, which occurred as a singleton. Moreover, spectra of the same strain that were matched to different OIUs are still located together in the network (Figure 1C) while this was not always the case in the dendrogram; e.g., OIU 9 and 19 corresponded to spectra of strain LMG 11495 and OIU 11 and 31 to spectra of strain LMG 11438. Importantly, in the network, the spectra of most strains appeared as well-separated, condensed clusters (Figure 1B). For a few strains, the distinction was less apparent because their clusters consisted of spectra of multiple strains (e.g., strains LMG 7761, LMG 11434 and LMG 11969).

\subsection{Network Analysis Differentiated OIUs Representing Closely Related Burkholderia Cepacia Complex Species}

We examined whether network analysis made it possible to differentiate references representing OIUs of genomically closely related strains and species. A set of 656 spectra derived from 21 strains belonging to six B. cepacia complex species were again taken from a previous study [15]. SPeDE dereplication of the 656 spectra resulted in 27 OIUs, which included at least one reference for $17 / 21$ strains and multiple references for 10 strains (Supplementary Table S3).

Hierarchical clustering analysis of these 27 references failed to group references by strain or species (Supplementary Figure S1). In contrast, network analysis based on the USF matrix distinguished nine well-separated clusters (Figure 2). The network nodes were color coded according to either their strain identity (Figure 2A) or their OIU classifier (Figure 2B). Seven clusters consisted of spectra derived from a single strain while clusters 1 and 7 contained spectra of multiple strains of B. multivorans (6/7 strains included) and B. cenocepacia (8/8 strains included), respectively (Figure 2A). Different references of the same strain consistently grouped in the same cluster as is illustrated by, e.g., OIU 149 and 150 of B. vietnamiensis R-67189 (Figure 2B, cluster 8), or by OIU 91 and OIU 92 of B. stabilis R-67113 (Figure 2B, cluster 4). For B. cenocepacia (cluster 7) a partitioning was observed for strains belonging to genomovar IIIA (pink variants) versus IIIB (green variants) (Figure 2A). Within cluster 1 , multiple references of the same strain lie closer to each other compared to references of other strains, i.e., R-67258 (turquoise) or R-68768 (orange) (Figure 2A, cluster 1). 
A

1. B. multivorans

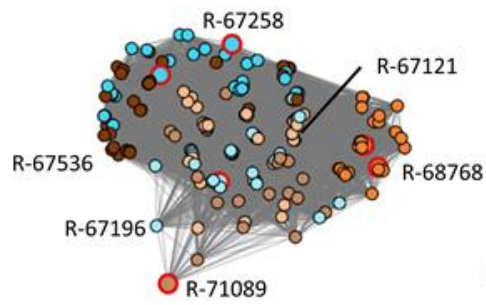

4. B. stabilis

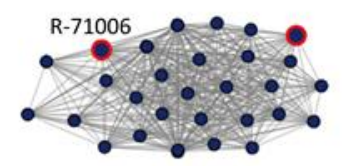

7. B. cenocepacia

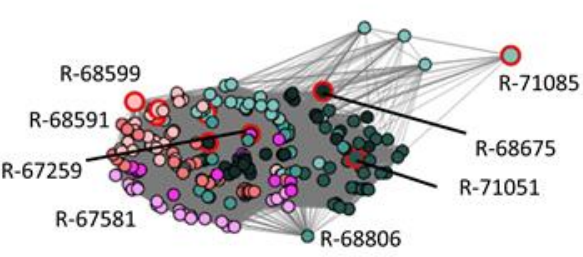

2. B. multivorans

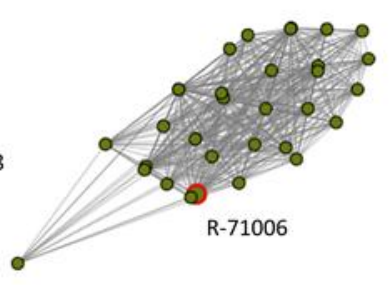

5. B. stabilis

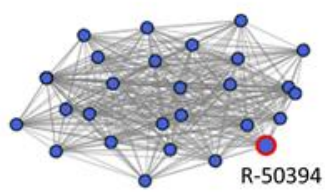

8. B. vietnamiensis

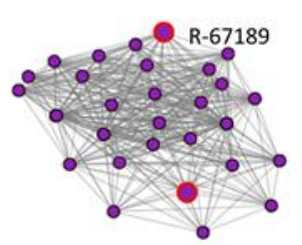

2.
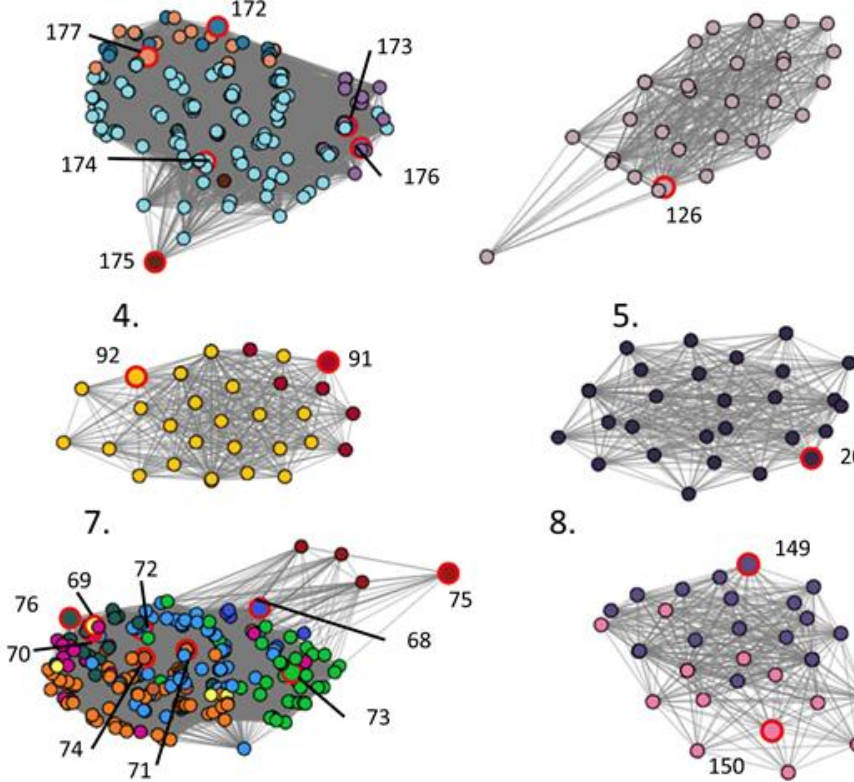

4.

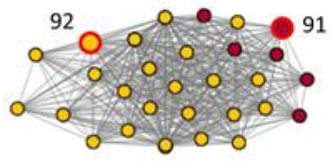

7.

1.

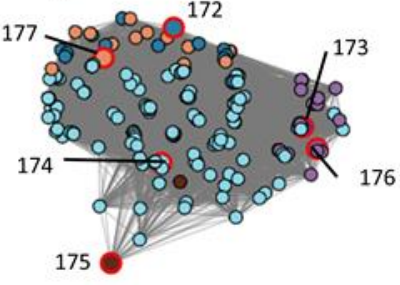

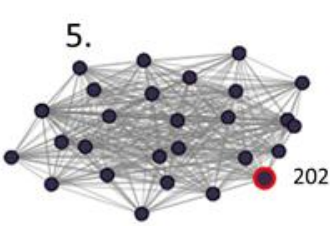

8.

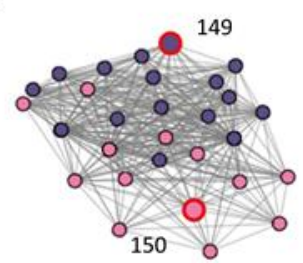

3. B. lata

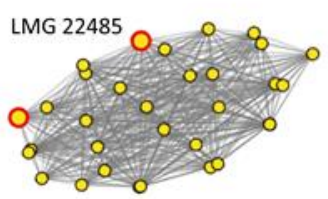

6. B. vietnamiensis

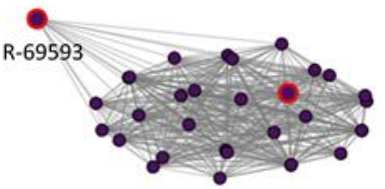

9. B. cepacia

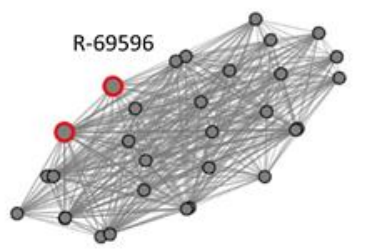

3.

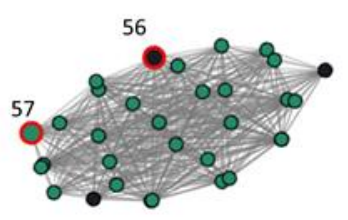

6.

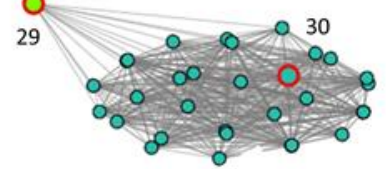

9.

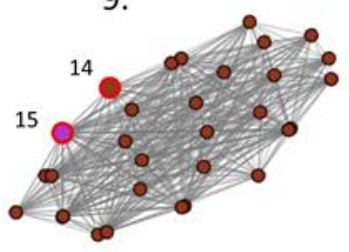

Figure 2. Network analysis on benchmark dataset containing 21 strains of six Burkholderia cepacia complex species. (A) Network analysis based on all zero USFs elements (edges) of the USF matrix, colored according to the strain number. (B) Network analysis based on all zero USFs elements (edges) of the USF matrix, colored accordingly to OIU number. The reference spectrum of each OIU is highlighted with large node sizes and red marking $(\mathbf{A}, \mathbf{B})$. 


\subsection{Dereplication of a Set of Soil Isolates}

A set of 200 spectra obtained from 134 soil isolates was selected based on their preliminary identification as Burkholderia sp. compared to the Bruker Biotyper DB5989 database or because the spectra comprised no USFs compared to some of the Burkholderia spectra. SPeDE dereplication of this dataset resulted in 43 OIUs, whereby the majority of the spectra $(75 \%)$ were matched to 16 references (Supplementary Table S4).

To determine how these 43 OIUs related to each other, a hierarchical clustering (Figure 3A) and network analysis was performed (Figure 3B). Based on the location of the references in the network, five condensed clusters of OIUs were apparent. These five clusters contained references of two (yellow), six (blue), eight (red), twelve (green) and sixteen (purple) OIUs, respectively. The OIU references 0 and 1 (yellow) were well separated from all others in the network and the dendrogram. The purple-colored references formed a single well-delineated cluster in the network analysis (Figure 3B), but not in the dendrogram (Figure 3A), where two purple subclusters were mixed with other references. Finally, the dendrogram failed to distinguish the remaining clusters of references that were apparent in the network analysis (i.e., the blue, red and green marked references).

A

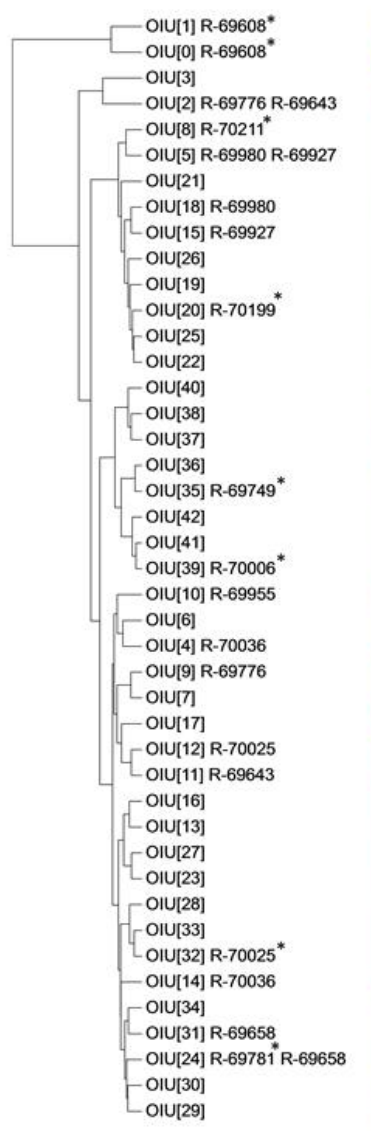

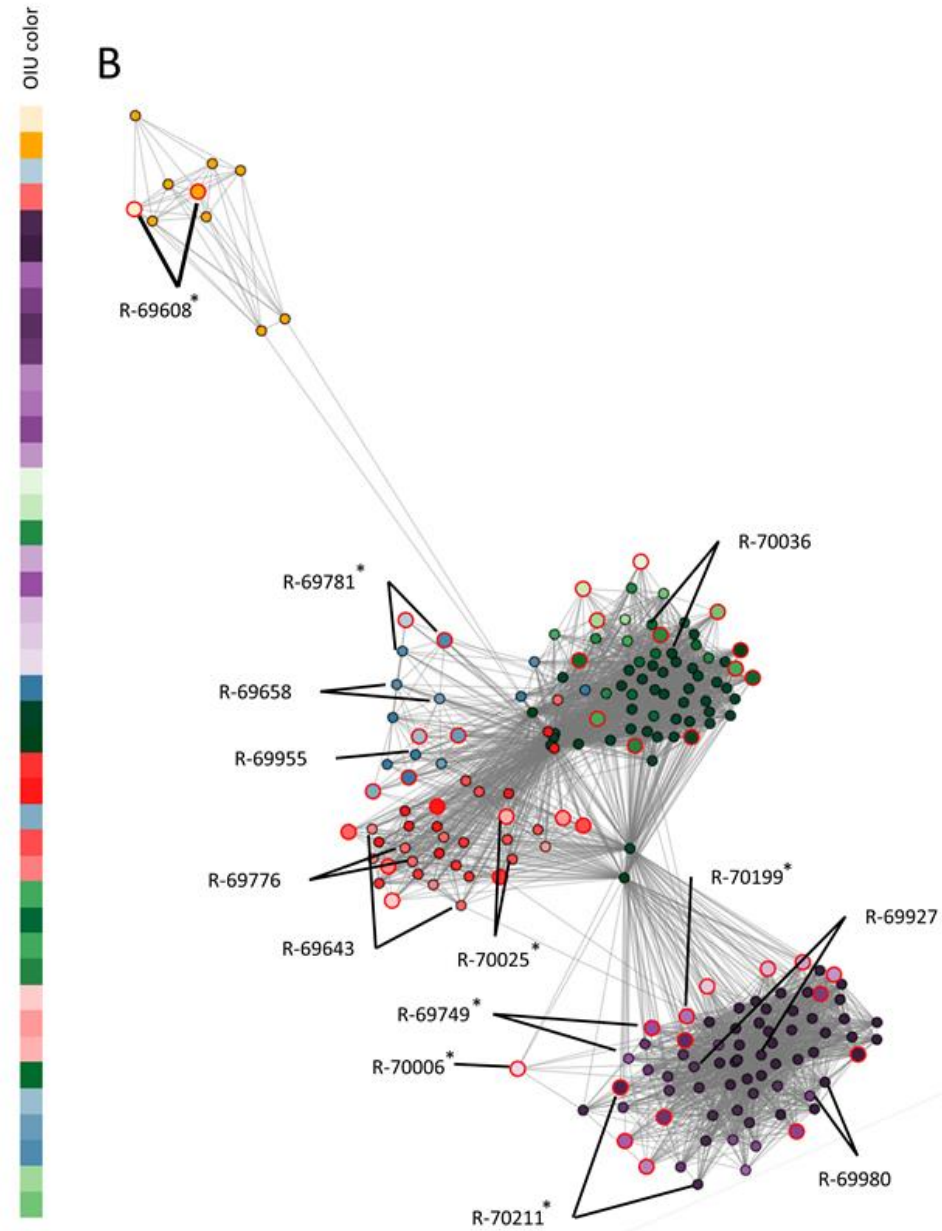

Figure 3. Comparison of hierarchical clustering versus network analysis on a case study dataset of 134 soil isolates. (A) Hierarchical clustering dendrogram of 43 reference spectra (UPGMA clustering algorithm) using the relative distance in the number of detected USFs. Aside from the corresponding OIU number, strains selected for further genomic analysis of which their spectra were matched to the OIU are indicated. (B) Network analysis based on all zero USFs elements (edges) of the USF matrix. Individual nodes are colored according to OIU number, as depicted in panel (A). The reference spectrum of each OIU is highlighted with large node sizes and red marking (B). The nodes of the spectra of each strain selected for further genomic analysis are marked by black lines $(\mathbf{B})$. Strains that were chosen as a reference are indicated with * $(\mathbf{A})$. 
To assess the value of hierarchical clustering and network analysis for further dereplication of $\mathrm{SPeDE}$ references, 14 isolates of which the spectra were assigned to 19 OIUs were selected for further genomic analysis (see below) based on the grouping of their references in both analyses (Figure 3A,B). Seven of these 14 isolates yielded a reference spectrum (R-69608, R-69749, R-69781, R-70006, R-70025, R-70199 and R-70211), while the other seven did not. Isolate R-69608 represented the yellow cluster as discussed above; isolates R-69749, R-69927, R-69980, R-70006, R-70199 and R-70211 represented the purple cluster; isolates R-69643, R-69776 and R-70025 represented the red cluster, isolates R-69655, R-69658 and $\mathrm{R}-69781$ represented the green cluster, and isolate R-70036 represented the blue cluster.

\subsection{Genomic Analysis of Soil Isolates}

Draft genome characteristics are provided in Supplementary Table S5. The genomewide ANI values among these fourteen isolates are summarized in Table 1. Based on the ANI cutoff of $95-96 \%$ routinely used for species delineation [27,28], the purple cluster isolates R-69749, R-69927, R-69980, R-70006, R-70199 and R-70211 which had a minimal pairwise ANI of about $99.5 \%$ belonged to a single species. The blue cluster isolates R-69658, R-69781 and R-69955 shared ANI values of approximately 100\% and exhibited ANI values of about $98 \%$ with the single green cluster isolate (R-70036), indicating that these four isolates represent a second species. The red cluster isolates R-69643, R-69776 and R-70025 also shared ANI values of at least $98 \%$ and exhibited ANI values of about $99 \%$ towards the single yellow cluster isolate R-69608, again indicating that these four isolates represent a single species. Isolates of the latter two species exhibited ANI values of about $95-96 \%$, which is precisely at the species delineation threshold $[27,28]$.

Table 1. Average nucleotide identity values between the soil isolates.

\begin{tabular}{|c|c|c|c|c|c|c|c|c|c|c|c|c|c|c|}
\hline & 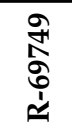 & 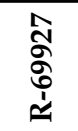 & $\begin{array}{l}\circ \\
80 \\
\text { ᄋ̆ } \\
\stackrel{1}{1}\end{array}$ & 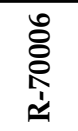 & $\begin{array}{l}\stackrel{2}{\sigma} \\
\stackrel{2}{\circ} \\
\stackrel{1}{1}\end{array}$ & 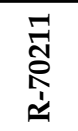 & 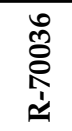 & 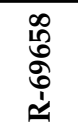 & 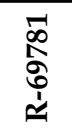 & 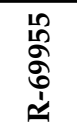 & 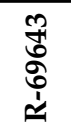 & $\begin{array}{l}\circ \\
\text { No } \\
\text { مٌ } \\
\stackrel{1}{1}\end{array}$ & 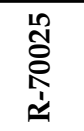 & $\begin{array}{l}\infty \\
\text { ठ̊. } \\
\text { రి } \\
\stackrel{1}{1}\end{array}$ \\
\hline R-69749 & 100 & 99.6 & 99.6 & 99.6 & 99.6 & 99.6 & 89.9 & 89.9 & 90.0 & 89.9 & 90.8 & 90.9 & 89.9 & 90.8 \\
\hline R-69927 & & 100 & 99.6 & 99.5 & 99.7 & 99.6 & 89.8 & 89.8 & 89.8 & 89.8 & 90.0 & 90.0 & 89.8 & 90.1 \\
\hline R-69980 & & & 100 & 99.6 & 99.6 & 99.7 & 89.9 & 90.0 & 90.0 & 90.0 & 90.1 & 90.0 & 90.0 & 90.0 \\
\hline R-70006 & & & & 100 & 99.7 & 99.7 & 89.8 & 89.9 & 89.8 & 89.9 & 90.1 & 90.0 & 89.9 & 90.1 \\
\hline R-70199 & & & & & 100 & 99.7 & 89.7 & 89.9 & 89.8 & 89.8 & 90.1 & 90.0 & 89.7 & 90.0 \\
\hline R-70211 & & & & & & 100 & 89.9 & 89.9 & 89.9 & 89.9 & 89.9 & 89.9 & 89.9 & 89.9 \\
\hline R-70036 & & & & & & & 100 & 98.0 & 98.0 & 98.0 & 95.3 & 95.3 & 95.1 & 95.3 \\
\hline R-69658 & & & & & & & & 100 & 100 & 100 & 95.6 & 95.6 & 95.4 & 95.5 \\
\hline R-69781 & & & & & & & & & 100 & 100 & 95.6 & 95.6 & 95.4 & 95.5 \\
\hline R-69955 & & & & & & & & & & 100 & 95.6 & 95.6 & 95.4 & 95.5 \\
\hline R-69643 & & & & & & & & & & & 100 & 99.0 & 98.7 & 99.0 \\
\hline R-69776 & & & & & & & & & & & & 100 & 98.8 & 99.0 \\
\hline R-70025 & & & & & & & & & & & & & 100 & 98.8 \\
\hline R-69608 & & & & & & & & & & & & & & 100 \\
\hline
\end{tabular}

\section{Discussion}

The resolution at which isolates can be categorized and differentiated is critical when selecting isolates for downstream analyses in large scale isolation campaigns. We previously introduced the SPeDE algorithm, which allows dereplication of MALDI-TOF MS spectral datasets into OIUs at an intraspecific level [15]. The assessment of taxonomic diversity using MALDI-TOF MS requires a high level of standardization to overcome reproducibility problems caused by biological and technical variation $[5,29,30]$. However, such variation is inevitably introduced into culturomics studies through the use of different isolation media, suboptimal growth conditions, the maximum number of isolates processed per day, sample preparation methodology and daily instrumental variation [14,30-32]. We previously showed that MALDI-TOF MS-based dereplication using the SPeDE algorithm 
is only moderately subject to sample variation, resulting in a limited overestimation of diversity [15]. However, the SPeDE algorithm does not provide a tool to estimate the degree of similarity between reference spectra to preferentially eliminate genomically redundant isolates for downstream analysis due to biological or technical sample variation [15].

In the present study, we compared the use of hierarchical clustering and network analysis as visualization tools of SPeDE's USF matrix data for this purpose. The hierarchical clustering dendrogram depicts the number of USFs between the references of each OIU as a distance. In contrast, the network analysis visualizes all comparisons for which no USFs were detected. In both the L. brevis (Figure 1) and the B. cepacia complex (Figure 2) datasets, the network analysis grouped all redundant references obtained from the same strain. This was to a much lesser extent the case when using hierarchical clustering (Figure 1 and Supplementary Figure S1), which confirmed earlier studies that reported that hierarchical clustering can fail to cluster and reliably separate biological replicate spectra of closely related strains $[18,19]$. In the B. cepacia complex dataset (Figure 2), distinct species consistently grouped in distinct network clusters. This was not the case in the hierarchical clustering dendrogram which again confirmed earlier reports [33-35].

The dereplication of spectra of 134 soil isolates yielded five visually defined clusters in the network consisting of 43 OIUs. These clusters did not correspond with clusters obtained through hierarchical clustering (Figure 3). Genomic analysis of fourteen isolates representing different clusters and OIUs showed that in the network the within-cluster genomic distance was maximally $0.8 \%$ ANI; the between-cluster genomic distances ranged from 89.7 to $99.0 \%$ ANI. The network analysis thus allowed to group the 43 OIUs into condensed clusters consisting of spectra of closely related isolates. Neither of the observed clusters comprised isolates representing distinct species, but isolates that represented the same species on two occasions grouped into distinct clusters.

Clearly, the three datasets indicated that network analysis resulted in a grouping of spectra that corresponded best with genomic relatedness of isolates and that network analysis also visualized the differentiation of OIUs by SPeDE at an infraspecific level; e.g., strains LMG 11993 and R-46486 in the L. brevis data set (Figure 1) and strain R-69608 in the soil isolate data set (Figure 3). Recently Giraud-Gatineau and colleagues reported that the potential of MALDI-TOF MS analysis to discriminate between infraspecific groups depends on the presence of so-called secondary sets of peaks [5]. The extent to which these secondary peak sets occur appears to be taxon dependent and may explain why in the present study USFs that allowed infraspecific differentiation were detected in some species only (Supplementary Table S3, Figure 2).

In conclusion, the results of the present study demonstrated that network visualization based on unique spectral features in MALDI-TOF mass spectra enabled a superior selection of genomically diverse operational isolation units compared to hierarchical clustering analysis. The network enabled efficient elimination of redundant SPeDE operational isolation units obtained from large-scale isolation campaigns of clinical or environmental samples. By selecting at least one OIU from each condensed cluster in the network the biological diversity within the analyzed dataset will be covered, which will effectively downsize the cost and time required for downstream genomic analyses.

Supplementary Materials: The following are available online at https:/ / www.mdpi.com/2076-260 7/9/2/416/s1, Figure S1: Hierarchical clustering analysis on benchmark dataset containing 21 strains of six Burkholderia species. Hierarchical clustering dendrogram of 27 reference spectra (UPGMA algorithm) using the relative distance in the number of detected USFs. Table S1: Overview of the datasets included in this study. Table S2: Results of SPeDE analysis of the L. brevis data set, Table S3: Results of the benchmark study for strains of the genus Burkholderia analyzed by SPeDE (from Dumolin et al., 2019); Table S4: Results of the soil isolate dataset; Table S5: Genomic statistics of draft genomes of strains of the case study dataset. 
Author Contributions: Conceptualization, C.D. and P.V.; methodology, C.D.; software, C.D.; formal analysis, C.D.; writing-original draft preparation, C.D.; writing-review and editing, C.D., C.P., E.D.C., N.B. and P.V.; visualization, C.D.; supervision, P.V.; Funding acquisition: N.B. and P.V. All authors have read and agreed to the published version of the manuscript.

Funding: C.D.: E.D.C.: N.B. and P.V. acknowledge the Geconcerteerde Onderzoeksactie (GOA) of Ghent University (BOF15/GOA/006) for funding this work. The research leading to results presented in this publication was carried out with infrastructure funded by EMBRC Belgium-FWO project GOH3817N.

Institutional Review Board Statement: Not applicable.

Informed Consent Statement: Not applicable.

Data Availability Statement: The data presented in this study are openly available in zenodo at [10.5281/zenodo.4055194].

Acknowledgments: We thank the Forest \& Nature Lab (ForNaLab) and K. Verheyen for providing us with a sample of the Gontrode Aelmoeseneie forest. We thank the Oxford Genomics Centre at the Wellcome Centre for Human Genetics (funded by Wellcome Trust grant reference 203141/Z/16/Z) for the generation and initial processing of the sequencing data.

Conflicts of Interest: The authors declare no conflict of interest. The funders had no role in the design of the study; in the collection, analyses, or interpretation of data; in the writing of the manuscript, or in the decision to publish the results.

\section{References}

1. De Carvalho, C.C.C.R. Whole cell biocatalysts: Essential workers from Nature to the industry. Microb. Biotechnol. 2017, 10, 250-263. [CrossRef]

2. BCC Research. Microbial Products: Technologies, Applications and Global Markets; Report Code: BIO086D; BCC Research: Wellesley, MA, USA, 2018.

3. Anteneh, Y.S.; Franco, C.M.M. Whole Cell Actinobacteria as Biocatalysts. Front. Microbiol. 2019, 10, 77. [CrossRef]

4. Zhang, L.; Sandrin, T.R. Maximizing the Taxonomic Resolution of MALDI-TOF-MS-Based Approaches to Bacterial Characterization: From Culture Conditions Through Data Analysis. In Applications of Mass Spectrometry in Microbiology; Springer International Publishing: Cham, Switzerland, 2016; pp. 147-181.

5. Giraud-Gatineau, A.; Texier, G.; Garnotel, E.; Raoult, D.; Chaudet, H. Insights Into Subspecies Discrimination Potentiality From Bacteria MALDI-TOF Mass Spectra by Using Data Mining and Diversity Studies. Front. Microbiol. 2020, 11, 1931. [CrossRef]

6. Lagier, J.-C.; Armougom, F.; Million, M.; Hugon, P.; Pagnier, I.; Robert, C.; Bittar, F.; Fournous, G.; Gimenez, G.; Maraninchi, M.; et al. Microbial culturomics: Paradigm shift in the human gut microbiome study. Clin. Microbiol. Infect. 2012, 18, $1185-1193$. [CrossRef]

7. Maier, T.; Klepel, S.; Renner, U.; Kostrzewa, M. Fast and reliable MALDI-TOF MS-based microorganism identification. Nat. Chem. Biol. 2006, 3. [CrossRef]

8. Dubois, D.; Grare, M.; Prere, M.-F.; Segonds, C.; Marty, N.; Oswald, E. Performances of the Vitek MS Matrix-Assisted Laser Desorption Ionization-Time of Flight Mass Spectrometry System for Rapid Identification of Bacteria in Routine Clinical Microbiology. J. Clin. Microbiol. 2012, 50, 2568-2576. [CrossRef] [PubMed]

9. Rahi, P.; Prakash, O.; Shouche, Y.S. Matrix-Assisted Laser Desorption/Ionization Time-of-Flight Mass-Spectrometry (MALDI-TOF MS) Based Microbial Identifications: Challenges and Scopes for Microbial Ecologists. Front. Microbiol. 2016, 7, 1359. [CrossRef] [PubMed]

10. Huschek, D.; Witzel, K. Rapid dereplication of microbial isolates using matrix-assisted laser desorption ionization time-of-flight mass spectrometry: A mini-review. J. Adv. Res. 2019, 19, 99-104. [CrossRef] [PubMed]

11. Kashtan, N.; Roggensack, S.E.; Rodrigue, S.; Thompson, J.W.; Biller, S.J.; Coe, A.; Ding, H.; Marttinen, P.; Malmstrom, R.R.; Stocker, R.; et al. Single-Cell Genomics Reveals Hundreds of Coexisting Subpopulations in Wild Prochlorococcus. Science 2014, 344, 416-420. [CrossRef] [PubMed]

12. Koeppel, A.F.; O Wertheim, J.; Barone, L.; Gentile, N.; Krizanc, D.; Cohan, F.M. Speedy speciation in a bacterial microcosm: New species can arise as frequently as adaptations within a species. ISME J. 2013, 7, 1080-1091. [CrossRef] [PubMed]

13. Spitaels, F.; Wieme, A.D.; Vandamme, P. MALDI-TOF MS as a Novel Tool for Dereplication and Characterization of Microbiota in Bacterial Diversity Studies. In Applications of Mass Spectrometry in Microbiology; Springer International Publishing: Cham, Switzerland, 2016; pp. 235-256.

14. Sandrin, T.R.; Goldstein, J.E.; Schumaker, S. MALDI TOF MS profiling of bacteria at the strain level: A review. Mass Spectrom. Rev. 2013, 32, 188-217. [CrossRef] 
15. Dumolin, C.; Aerts, M.; Verheyde, B.; Schellaert, S.; Vandamme, T.; Van Der Jeugt, F.; De Canck, E.; Cnockaert, M.; Wieme, A.D.; Cleenwerck, I.; et al. Introducing SPeDE: High-Throughput Dereplication and Accurate Determination of Microbial Diversity from Matrix-Assisted Laser Desorption-Ionization Time of Flight Mass Spectrometry Data. mSystems 2019, 4, e00437-19. [CrossRef] [PubMed]

16. Christner, M.; Dressler, D.; Andrian, M.; Reule, C.; Petrini, O. Identification of Shiga-Toxigenic Escherichia coli outbreak isolates by a novel data analysis tool after matrix-assisted laser desorption/ionization time-of-flight mass spectrometry. PLoS ONE 2017, 12, e0182962. [CrossRef]

17. Ghyselinck, J.; Van Hoorde, K.; Hoste, B.; Heylen, K.; De Vos, P. Evaluation of MALDI-TOF MS as a tool for high-throughput dereplication. J. Microbiol. Methods 2011, 86, 327-336. [CrossRef] [PubMed]

18. Strejcek, M.; Smrhova, T.; Junkova, P.; Uhlik, O. Whole-Cell MALDI-TOF MS Versus $16 S$ rRNA Gene Analysis for Identification and Dereplication of Recurrent Bacterial Isolates. Front. Microbiol. 2018, 9, 1294. [CrossRef] [PubMed]

19. Oberle, M.; Wohlwend, N.; Jonas, D.; Maurer, F.P.; Jost, G.; Tschudin-Sutter, S.; Vranckx, K.; Egli, A. The Technical and Biological Reproducibility of Matrix-Assisted Laser Desorption Ionization-Time of Flight Mass Spectrometry (MALDI-TOF MS) Based Typing: Employment of Bioinformatics in a Multicenter Study. PLoS ONE 2016, 11, e0164260. [CrossRef] [PubMed]

20. Purves, K.; Macintyre, L.; Brennan, D.; Hreggviðsson, G.; Kuttner, E. Using Molecular Networking for Microbial Secondary Metabolite Bioprospecting. Metabolites 2016, 6, 2. [CrossRef] [PubMed]

21. Yang, J.Y.; Sanchez, L.M.; Rath, C.M.; Liu, X.; Boudreau, P.D.; Bruns, N.; Glukhov, E.; Wodtke, A.; De Felicio, R.; Fenner, A.; et al. Molecular Networking as a Dereplication Strategy. J. Nat. Prod. 2013, 76, 1686-1699. [CrossRef] [PubMed]

22. Vanhellemont, M.; Verheyen, K.; Baeten, L. Relating changes in understorey diversity to environmental drivers in an ancient forest in northern Belgium. Plant Ecol. Evol. 2014, 147, 22-32. [CrossRef]

23. Letunic, I.; Bork, P. Interactive Tree Of Life (iTOL) v4: Recent updates and new developments. Nucleic Acids Res. 2019, 47, W256-W259. [CrossRef]

24. Hagberg, A.A.; Schult, D.A.; Swart, P.J. Exploring Network Structure, Dynamics, and Function using NetworkX. In Proceedings of the 7th Python in Science Conference, Pasadena, CA, USA, 19-24 August 2008; pp. 11-15.

25. Bokeh Development Team. Bokeh: Python Library for Interactive Visualization. Available online: https://bokeh.pydata.org/en/ latest/ (accessed on 1 December 2020).

26. Gurevich, A.; Saveliev, V.; Vyahhi, N.; Tesler, G. QUAST: Quality assessment tool for genome assemblies. Bioinformatics 2013, 29, 1072-1075. [CrossRef] [PubMed]

27. Lee, I.; Kim, Y.O.; Park, S.-C.; Chun, J. OrthoANI: An improved algorithm and software for calculating average nucleotide identity. Int. J. Syst. Evol. Microbiol. 2016, 66, 1100-1103. [CrossRef] [PubMed]

28. Goris, J.; Konstantinidis, K.T.; Klappenbach, J.A.; Coenye, T.; Vandamme, P.; Tiedje, J.M. DNA-DNA hybridization values and their relationship to whole-genome sequence similarities. Int. J. Syst. Evol. Microbiol. 2007, 57, 81-91. [CrossRef]

29. Arnold, R.J.; Karty, J.A.; Ellington, A.D.; Reilly, J.P. Monitoring the growth of a bacteria culture by MALDI-MS of whole cells. Anal. Chem. 1999, 71, 1990-1996. [CrossRef]

30. Ruelle, V.; El Moualij, B.; Zorzi, W.; Ledent, P.; De Pauw, E. Rapid identification of environmental bacterial strains by matrixassisted laser desorption/ionization time-of-flight mass spectrometry. Rapid Commun. Mass Spectrom. 2004, 18, $2013-2019$. [CrossRef]

31. Giebel, R.; Worden, C.; Rust, S.; Kleinheinz, G.; Robbins, M.; Sandrin, T. Microbial Fingerprinting using Matrix-Assisted Laser Desorption Ionization Time-Of-Flight Mass Spectrometry (MALDI-TOF MS). Adv. Appl. Microbiol. 2010, 71, 149-184. [PubMed]

32. Wieme, A.D.; Spitaels, F.; Aerts, M.; De Bruyne, K.; Van Landschoot, A.; Vandamme, P. Effects of Growth Medium on MatrixAssisted Laser Desorption-Ionization Time of Flight Mass Spectra: A Case Study of Acetic Acid Bacteria. Appl. Environ. Microbiol. 2014, 80, 1528-1538. [CrossRef]

33. Christensen, J.J.; Dargis, R.; Hammer, M.; Justesen, U.S.; Nielsen, X.C.; Kemp, M. The Danish MALDI-TOF MS Study Group Matrix-Assisted Laser Desorption Ionization-Time of Flight Mass Spectrometry Analysis of Gram-Positive, Catalase-Negative Cocci Not Belonging to the Streptococcus or Enterococcus Genus and Benefits of Database Extension. J. Clin. Microbiol. 2012, 50, 1787-1791. [CrossRef] [PubMed]

34. Stafsnes, M.H.; Dybwad, M.; Brunsvik, A.; Bruheim, P. Large scale MALDI-TOF MS based taxa identification to identify novel pigment producers in a marine bacterial culture collection. Antonie Leeuwenhoek 2012, 103, 603-615. [CrossRef]

35. Wang, J.; Zhou, N.; Xu, B.; Hao, H.; Kang, L.; Zheng, Y.; Jiang, Y.; Jiang, H. Identification and Cluster Analysis of Streptococcus pyogenes by MALDI-TOF Mass Spectrometry. PLoS ONE 2012, 7, e47152. [CrossRef] [PubMed] 